Yipsy Roque Benito

Modelagem da produção simultânea de frio, calor e energia elétrica

Dissertação de Mestrado

Dissertação apresentada como requisito parcial para obtenção do título de Mestre pelo Programa de PósGraduação em Engenharia Mecânica da PUC-Rio.

ncia térmica vs carga para um grupo gerador/turbina a gás operando com Orientadores: José Alberto dos Reis Parise José Viriato Coelho Vargas

Rio de Janeiro, Abril de 2007 
Yipsy Roque Benito

\title{
Modelagem da produção simultânea de frio, calor e energia elétrica
}

\begin{abstract}
Dissertação apresentada como requisito parcial para obtenção do título de Mestre pelo Programa de PósGraduação em Engenharia Mecânica da PUC-Rio. Aprovada pela Comissão Examinadora abaixo assinada.
\end{abstract}

José Alberto dos Reis Parise

Orientador

Departamento de Engenharia Mecânica - PUC-Rio

José Viriato Coelho Vargas

Co-Orientador

Departamento de Engenharia Mecânica - UFPR

Sergio Leal Braga

Departamento de Engenharia Mecânica - PUC-Rio

Carlos Valois Maciel Braga

Departamento de Engenharia Mecânica - PUC-Rio

Nisio de Carvalho Lobo Brum

Departamento de Engenharia Mecânica - UFRJ

José Eugenio Leal

Coordenador(a) Setorial do Centro Técnico Científico - PUC-Rio

Rio de Janeiro, 27 de abril de 2007 
Todos os direitos reservados. É proibida a reprodução total ou parcial do trabalho sem autorização da universidade, da autora e do orientador.

\section{Yipsy Roque Benito}

Graduou-se em Engenharia Mecânica na Universidad Central "Marta Abreu" de Las Villas, Cuba, em 1998. Atua na área de Engenharia Mecânica, com ênfase em Refrigeração e Aproveitamento da Energia. Tem trabalhado em análises de sistemas energéticos.

Ficha Catalográfica

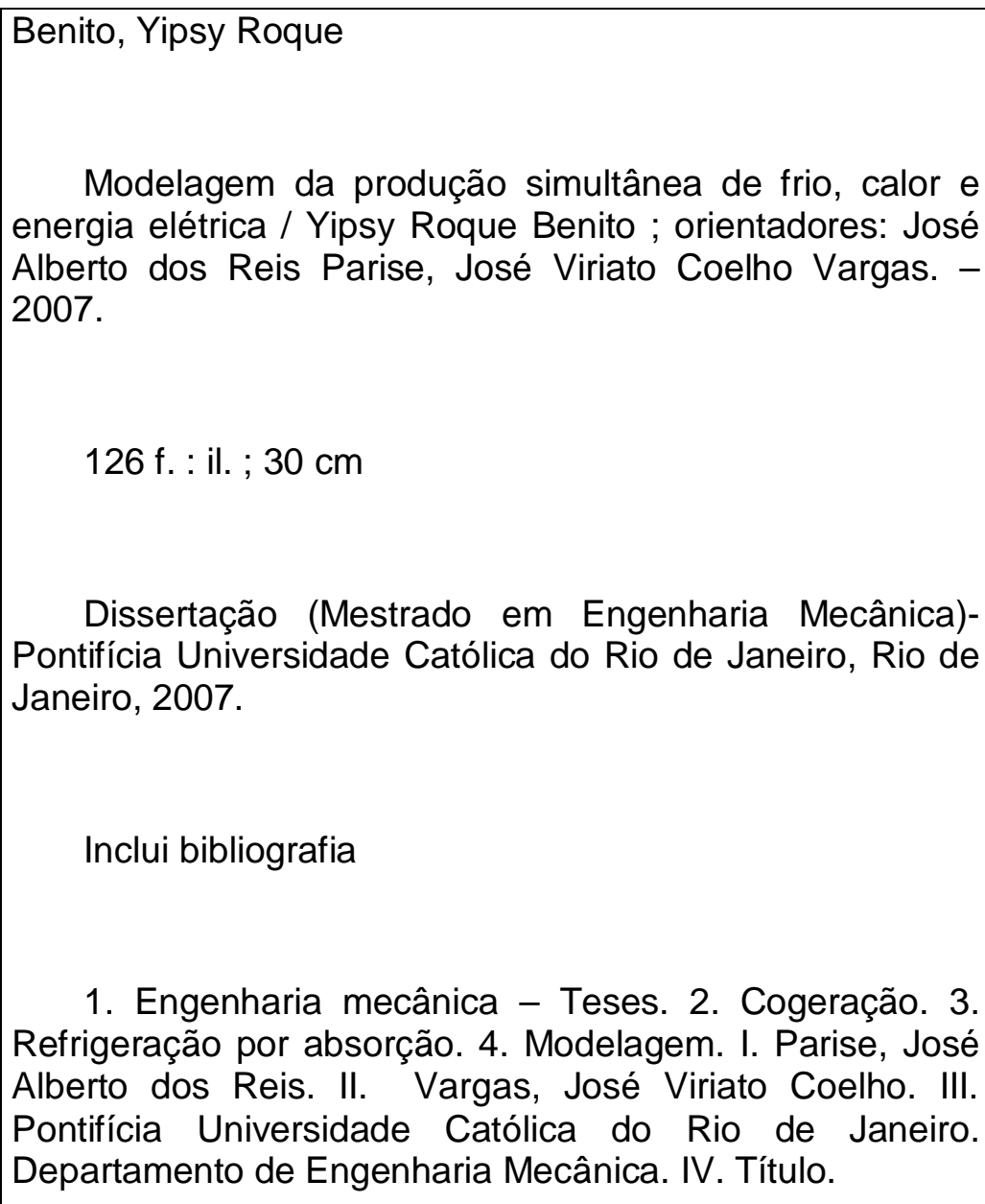

Modelagem da produção simultânea de frio, calor e energia elétrica / Yipsy Roque Benito ; orientadores: José Alberto dos Reis Parise, José Viriato Coelho Vargas. 2007.

126 f. : il. ; $30 \mathrm{~cm}$

Dissertação (Mestrado em Engenharia Mecânica)Pontifícia Universidade Católica do Rio de Janeiro, Rio de Janeiro, 2007.

\section{Inclui bibliografia}

1. Engenharia mecânica - Teses. 2. Cogeração. 3. Refrigeração por absorção. 4. Modelagem. I. Parise, José Alberto dos Reis. II. Vargas, José Viriato Coelho. III. Pontifícia Universidade Católica do Rio de Janeiro. Departamento de Engenharia Mecânica. IV. Título. 


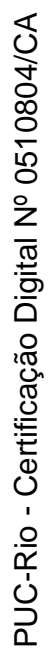

Para meus pais, Margot e Pablo 


\section{Agradecimentos}

Ao meu orientador, Prof. José Alberto dos Reis Parise, pela dedicação, pela força e por ter confiado na minha capacidade.

Ao meu co-orientador, Prof. José Viriato Coelho Vargas, pelo apoio e incentivo.

Ao Prof. Sandro Bastos Ferreira, pelas oportunas observações.

Ao Ricardo Hernandez Pereira e Marcus Pereira, pela disponibilização dos dados experimentais.

À Agência Nacional de Petróleo (ANP) e à Financiadora de Estudos e Projetos (FINEP), pelo apoio financeiro através do Programa de Recursos Humanos da ANP para o Sector Petróleo e Gás (PRH-ANP/MCT).

Às agencias CNPq e FAPERJ, pelo apoio financeiro ao projeto.

Ao grupo NILKO, pelo apoio financeiro.

Aos colegas e amigos da Pós-graduação, Frank, Ysrael, Juliana, Mayra, Ana Luíza, Germaín, Minchola, Epifánio, e Diogo, pela presença nos grandes e pequenos momentos.

À Rosely, Grace e Carolina, do Departamento de Engenharia Mecânica da PUCRio, pela ajuda inestimável.

Ao Freddy, Alejandro e Lisbet, que seguem aqui, apesar da distância.

À minha irmã, Xenia.

E ao Pedro Rocha de Oliveira, por muitas coisas. 


\section{Resumo}

Benito, Yipsy Roque; Parise, José Alberto dos Reis. Modelagem da produção simultânea de frio, calor e energia elétrica. Rio de Janeiro, 2007. 126p. Dissertação de Mestrado - Departamento de Engenharia Mecânica, Pontifícia Universidade Católica do Rio de Janeiro.

O presente trabalho divide-se em dois estudos: uma análise global de sistemas de cogeração e uma modelagem de um ciclo de refrigeração por absorção. O primeiro estudo apresenta as equações dos balanços energéticos de um sistema de cogeração operando com dois motores térmicos distintos (turbina a gás e motor de combustão interna, ambos utilizando diesel como combustível). Para a produção de frio emprega-se um "chiller" de absorção, acionado a partir de calor de rejeito dos motores, e outro de compressão de vapor auxiliar. As equações de balanço de energia e de exergia, aplicadas a cada componente, formam um sistema não linear de equações que, resolvido, fornece o desempenho do sistema para diferentes condições de operação. O segundo estudo é parte de um projeto mais abrangente, destinado a desenvolver a tecnologia de um sistema de refrigeração por absorção. É apresentado o modelo matemático que caracteriza uma instalação de pequeno porte operando com uma mistura água-amônia. Foram aplicadas as equações de conservação de massa e energia para cada componente do ciclo, determinadas as propriedades termodinâmicas em cada ponto do ciclo e aplicadas hipóteses simplificadoras de modo a descrever matematicamente os processos físicos envolvidos. O modelo resultante foi aplicado a um sistema existente. A comparação entre os resultados previstos pelo modelo e os obtidos experimentalmente foi satisfatória. Uma vez implementados os modelos de ambos os estudos, realizaram-se simulações para casos particulares de operação, possibilitando a verificação da influência das principais variáveis sobre o desempenho dos sistemas analisados. Na solução dos modelos matemáticos foi utilizado o software EES ${ }^{\circledR}$.

\section{Palavras-chave}

Cogeração, refrigeração por absorção, modelagem 


\section{Abstract}

Benito, Yipsy Roque; Parise, José Alberto dos Reis. Modeling of offshore production of cold, heat and electrical power. Rio de Janeiro, 2007. 126p. MSc. Dissertation - Departamento de Engenharia Mecânica, Pontifícia Universidade Católica do Rio de Janeiro.

The present work embodies two studies: a global analysis of cogeneration systems and an absorption refrigeration cycle model. The first study presents the main equations for energy and exergy balances of a cogeneration system operating with two distinct prime movers (a gas turbine and an internal combustion engine, both powered by diesel oil). For cooling production, an absorption chiller, driven by the prime movers' waste heat, and an auxiliary vapor compression chiller are employed. The energy and exergy balance equations were applied to each component of the system, composing a non-linear system of algebraic equations whose solution provides the performance of the system under different operating conditions. The second study is part of a broader project aiming at the development of an absorption refrigeration system. A mathematical model is presented, describing a small-size absorption refrigeration installation employing a water-ammonia mixture as working fluid. Mass and energy conservation equations were applied to each component of the cycle. Thermodynamic properties of the working fluids were calculated at each point of the cycle. Also, simplifying assumptions were applied. The resulting model was applied to simulate the behavior of an existing system. Comparison of predicted results with experimental data was satisfactory. A parametric analysis was also carried out with the simulation model. The models of both studies were implemented in ESS ${ }^{\circledR}$ software. Simulations were carried out so as to analyze the systems under particular operational conditions and to assess the influence of the main variables on system performance.

\section{Keywords}

Cogeneration, absorption refrigeration, modeling. 


\section{Sumário}

1 Introdução 19

$\begin{array}{ll}1.1 \text { Contexto geral } & 19\end{array}$

1.2 Preâmbulo $\quad 20$

1.2.1 Demandas simultâneas de refrigeração e climatização, energia elétrica e aquecimento 20

1.2.2 Projeto DORAGEX 21

1.3 Objetivos 22

$\begin{array}{ll}1.4 \text { Organização do trabalho } & 23\end{array}$

2 Estudo termodinâmico de um sistema de cogeração operando com dois motores térmicos distintos. 24

2.1 Introdução $\quad 24$

$\begin{array}{ll}2.1 .1 \text { Objetivos do capítulo } & 27\end{array}$

2.2 Descrição do sistema $\quad 27$

2.3 Modelo Termodinâmico 30

2.3.1 Hipóteses simplificadoras $\quad 30$

2.3.2 Definições 31

2.3.3 Determinações da exergia 32

2.3.4 Balanço de energia nos motores térmicos $\quad 34$

2.3.5 Contabilidade de exergia 34

2.3.5.1 Turbina a gás $\quad 35$

2.3.5.2 Motor de combustão interna $\quad 35$

2.3.5.3 Chiller de absorção 36

2.3.5.4 Chiller de compressão de vapor $\quad 36$

$\begin{array}{ll}\text { 2.3.5.5 Balanço global da exergia da planta } & 37\end{array}$

2.3.6 Solução $\quad 37$

2.4 Análise paramétrica 38

2.4.1 Condições de operação 38

2.4.2 Valores numéricos adotados $\quad 39$

2.4.3 Caso \#1: Demanda de frio igual a produção do chiller de absorção, $\mathcal{Q}_{C, A C}=\mathcal{Q}_{C, D E}$

2.4.4 Caso \#2: Produção de frio excede a demanda, $\boldsymbol{Q}_{C, A C}>\boldsymbol{Q}_{C, D E}$

2.4.5 Caso \#3: Demanda de frio excede a produção frigorífica do chiller de absorção, $\mathcal{Q}_{c, A C}<\mathcal{Q}_{c, D E} \quad 55$

3 Modelagem da refrigeração por absorção 62 
3.1 Introdução $\quad 62$

3.1.1 Justificativa 63

3.1.2 Objetivos 64

3.2 Revisão Bibliográfica $\quad 65$

3.3 Descrição do sistema de refrigeração por absorção 68

3.3.1 Ciclo termodinâmico. $\quad 69$

3.4 Modelo matemático $\quad 74$

3.4.1 Hipóteses simplificadoras $\quad 75$

3.4.2 Determinações das pressões $\quad 76$

3.4.2.1 Pressão de condensação 76

$\begin{array}{ll}\text { 3.4.2.2 Pressão de evaporação } & 77\end{array}$

$\begin{array}{ll}\text { 3.4.2.3 Pressão intermediária } & 77\end{array}$

3.4.3 Balanços de energia, massa e espécies dos volumes de controle $\quad 79$

3.4.3.1 Ciclo de amônia $\quad 79$

3.4.3.1.1 Condensador $\quad 80$

3.4.3.1.2 Evaporador $\quad 81$

3.4.3.1.3 Regenerador interno $\quad 83$

3.4.3.1.4 Dispositivos de expansão $\quad 85$

3.4.3.2 Compressor térmico $\quad 87$

$\begin{array}{ll}\text { 3.4.3.2.1 Processo de absorção } & 87\end{array}$

3.4.3.2.1.1 Absorvedor-regenerador ou pré-absorvedor 88

3.4.3.2.1.2 Absorvedor resfriado por água $\quad 91$

3.4.3.2.2 A bomba da solução 92

3.4.3.2.3 Retificador 93

3.4.3.2.4 Gerador $\quad 96$

3.4.3.2.5 Dispositivo de expansão do compressor térmico 100

3.5 Solução 101

$\begin{array}{ll}3.6 \text { Simulação } & 101\end{array}$

3.6.1 Principais aproximações e constantes da simulação 103

3.6.2 Comparação com dados experimentais 104

$\begin{array}{ll}3.7 \text { Análise exergética } & 108\end{array}$

$\begin{array}{ll}\text { 3.7.1 Determinação da exergia } & 108\end{array}$

$\begin{array}{ll}\text { 3.7.2 Contabilidade de exergia } & 110\end{array}$

3.8 Análise de indicadores do desempenho do sistema 110

3.9 Análise paramétrica 111

4 Conclusões e recomendações para trabalhos futuros $\quad 117$

4.1 Conclusões 117

4.2 Recomendações para trabalhos futuros 119

5 Referências Bibliográficas $\quad 121$ 


\section{Lista de figuras}

Figura 1. Esquema geral da planta para geração de potencia elétrica e produção de frio

Figura 2. Eficiência térmica vs carga para um grupo gerador/turbina a gás operando com gás natural e com controle da carga parcial através da regulação da temperatura de entrada à turbina (Ferreira, 2002)

Figura 3. Eficiência térmica vs carga para um grupo gerador/motor de combustão interna operando com óleo Diesel. Pontos experimentais de Pereira (2006a)

Figura 4. Efeito da temperatura no COP do chiller de absorção (Colonna e Gabrielli, 2006)

Figura 5. Esquema da instalação para o caso \#1. $\mathcal{Q}_{c, A C}=\mathcal{Q}_{c, D E}$

Figura 6. Razão de carga da turbina, $\chi_{G T}$ em função da razão de demanda elétrica, $\gamma_{W}$

Figura 7. Caso \#1. Variação da produção de frio em função de $\lambda$ para diferentes valores de demanda elétrica

Figura 8. Caso \#1. Desempenhos globais e dos motores térmicos com a variação de $\lambda$, para $\gamma_{W}=1,0$

Figura 9. Caso \#1. Comportamento de $E C R$ e $\psi_{G B}$ para $\gamma_{W}=1,0$

Figura 10. Caso \#1.Variação da razão de conversão de energia com $\lambda$

Figura 11. Caso \#1. Variação da eficiência racional da planta $\operatorname{com} \lambda$

Figura 12. Esquema da instalação para o caso \#2

Figura 13. Comparação da razão de conversão de energia do caso \# 2 com a do caso \# 1 para $\gamma_{W}=1,0$.

Figura 14. Comparação da eficiência racional do caso \# 2 com a do caso \# 1 para

$$
\gamma_{W}=1,0
$$


Figura 15a. Caso \# 2. Variação de $E C R, \psi_{G B}$ e $\eta \operatorname{com} \lambda$, para $R=2$ e $\gamma_{W}=1,0$

Figura 15b. Caso \#2. Comportamento de $E C R, \psi_{G B}$ e $\eta \operatorname{com} \lambda$, para $R=2$ e

$$
\gamma_{W}=0,5
$$

Figura 16a. Caso \#2. Comportamento de $E C R$ com $R$ para $\gamma_{W}=1,0$

Figura16b. Caso \#2. Comportamento de $E C R$ com $R$ para $\gamma_{W}=0,5$

Figura 17a. Caso \#2. Comportamento de $\psi_{G B} \operatorname{com} R$, para $\gamma_{W}=1,0$

Figura 17b. Caso \#2. Comportamento de $\psi_{G B} \operatorname{com} R$, para $\gamma_{W}=0,5$

Figura 18a. Caso \#2. Comportamento das eficiências racionais nos diferentes subsistemas da planta, para $\gamma_{W}=1,0$ e $R=2$

Figura 18b. Caso \#2. Comportamento das eficiências racionais nos diferentes subsistemas da planta, para $\gamma_{W}=0,5$ e $R=2$

Figura 19a. Caso \#2. Distribuição da exergia do combustível de entrada à planta em produtos, perdas e destruição $\left(\gamma_{W}=1,0\right.$ e $\left.R=2\right)$

Figura 19b. Caso \#2. Distribuição da exergia do combustível de entrada à planta em produtos, perdas e destruição $\left(\gamma_{W}=0,5\right.$ e $\left.R=2\right)$

Figura 20. Esquema da instalação para o caso \# 3

Figura 21. Comparação da razão de conversão de energia do Caso \# 3 com a do Caso\#1 para $\gamma_{W}=1,0$

Figura 22. Comparação da eficiência racional do caso \# 3 com a do caso \# 1 para

$$
\gamma_{W}=1,0
$$

Figura 23a. Caso \#3. Comportamento de $E C R, \psi_{G B}$ e $\eta \operatorname{com} \lambda$, para $\gamma_{W}=1,0$ e $R=0,5$

Figura 23b. Caso \#3. Comportamento de $E C R, \psi_{G B}$ e $\eta \operatorname{com} \lambda$, para $\gamma_{W}=0,5 \mathrm{e}$ 


$$
R=0,5
$$

Figura 24a. Caso \# 3. Desempenho global para $\lambda=0,6$ e $\gamma_{W}=1,0$

Figura 24b. Caso \# 3. Desempenho global para $\lambda=0,6$ e $\gamma_{W}=0,5$

Figura 25a. Caso \# 3. Distribuição da exergia do combustível de entrada à planta em produtos, perdas e destruição $\left(R=0,5\right.$ e $\left.\gamma_{W}=1,0\right)$

Figura 25b. Caso \# 3. Distribuição da exergia do combustível de entrada à planta em produtos, perdas e destruição $\left(R=0,5\right.$ e $\left.\gamma_{W}=0,5\right)$

Figura 26a. Caso \#3. Distribuição da exergia do combustível de entrada à planta em produtos, perdas e destruição $\left(\lambda=0,6\right.$ e $\left.\gamma_{W}=1,0\right)$

Figura 26b. Caso\#3. Distribuição da exergia do combustível de entrada à planta em produtos, perdas e destruição $\left(\lambda=0,6\right.$ e $\left.\gamma_{W}=0,5\right)$

Figura 26c. Caso\#3. Distribuição da exergia do combustível de entrada à planta em produtos, perdas e destruição $\left(\lambda=0,6\right.$ e $\left.\gamma_{W}=0,4\right)$

Figura 27. Ciclo básico de absorção

Figura 28. Instalação do refrigerador por absorção na UFPR

Figura 29. ROBUR. Esquema do modelo Robur GAHP-W operando de acordo com o ciclo de absorção de vapor. Fonte Robur, (2005)

Figura 30. Esquema $\mathrm{P}$ - T do ciclo de absorção de vapor com pré-aquecimento da solução forte no retificador e recirculação no pré-absorvedor

Figura 31. Plano h-x para a mistura amônia-água 75

Figura 32. Volume de controle do Condensador 80

Figura 33. Volume de controle do Evaporador 82

Figura 34. Regenerador interno $\quad 84$

Figura 35. Dispositivos de expansão I e II 86

$\begin{array}{ll}\text { Figura 36. Absorvedor regenerativo } & 88\end{array}$

Figura 37. Mistura adiabática pelo lado da carcaça 89

Figura 38. Representação esquemática dos processos de absorção no diagrama h- 
Figura 39. Absorvedor resfriado por água

$\begin{array}{ll}\text { Figura 40. Bomba da solução } & 93\end{array}$

$\begin{array}{lr}\text { Figura 41. Resfriador de refluxo } & 94\end{array}$

Figura 42. Processo de troca de calor prévio à troca de massa 94

Figura 43. Representação esquemática do processo de retificação no diagrama h-x 95

Figura 44 Volume de controle do gerador 96

Figura 45 Gerador. Volumes de controle internos $\quad 97$

Figura 46. Representação esquemática do processo de geração no diagrama h-x 99

Figura 47. Detalhe do diagrama h-x para a mistura água-amônia na região de altas pressões para concentrações próximas a 1

Figura 48. Comparação das temperaturas de saída da solução gelada de águaetilenoglicol com dados experimentais

Figura 49. Comparação das temperaturas de saída da água quente com dados experimentais

Figura 50. Comparação entre as taxas de refrigeração e de rejeito previstas e experimentais

Figura 51. Potência frigorífica em função da vazão de solução gelada para diferentes vazões de água quente

Figura 52. Potência térmica de rejeito em função da vazão de água quente para diferentes vazões de solução gelada

Figura 53. Pressão de condensação em função da vazão de água quente. $T_{h w ; i n}=295 \mathrm{~K} ; T_{c w ; i n}=288 \mathrm{~K}, n \alpha_{c w}=0,37 \mathrm{~kg} / \mathrm{s}$

Figura 54. Pressão de evaporação em função da vazão de solução fria. $T_{h w ; i n}=295 \mathrm{~K} ; T_{c w ; i n}=288 \mathrm{~K} n \hat{k}_{h w}=0,40 \mathrm{~kg} / \mathrm{s}$

Figura 55. Potência frigorífica versus vazão de solução fria para $T_{h w ; i n}=295 \mathrm{~K} ; T_{c w ; i n}=288 \mathrm{~K}$

Figura 56. Temperatura de saída da água quente versus vazão de água quente para $T_{h w ; i n}=295 \mathrm{~K} ; T_{c w ; i n}=288 \mathrm{~K}$ e para diferentes vazões de solução gelada

Figura 57. Taxa de fornecimento de calor no gerador em função da vazão de solução gelada para vários valores da vazão de água quente para $T_{h w ; i n}=295 \mathrm{~K} ; T_{c w ; i n}=288 \mathrm{~K}$

Figura 58. Desempenho do sistema para $h \alpha_{h w}=0,5 \mathrm{~kg} / \mathrm{s} ; \quad T_{h w ; i n}=295 K$;

$$
T_{c w ; i n}=288 \mathrm{~K}
$$


Figura 59. Temperaturas de saída da água quente e da solução fria em função da vazão da solução fria para $n \kappa_{h w}=0,5 \quad \mathrm{~kg} / \mathrm{s}$,
$T_{h w ; i n}=295 \mathrm{~K} ; T_{c w ; i n}=288 \mathrm{~K}$

Figura 60. Vazão de refrigerante em função da vazão de água quente. $T_{h w ; i n}=295 \mathrm{~K} ; T_{c w ; i n}=288 \mathrm{~K}$ e ${ }_{n} \alpha_{c w}=0,37 \mathrm{~kg} / \mathrm{s}$

Figura 61. Efetividade do absorvedor esfriado por água em função da vazão de água quente, para diferentes vazões de solução fria. $T_{h w ; i n}=295 \mathrm{~K} ; T_{c w ; i n}=288 \mathrm{~K}$

\section{Lista de tabelas}

Tabela 1. Dados obtidos a partir dos casos analisados por Colonna e Gabrielli (2006)

Tabela 2. Dados nominais do Robur, modelo GAHP-W (Robur, 2005).

Tabela 3. Estados termodinâmicos do sistema. 


\section{Simbologia}

a Coeficiente dependente do grau de sub-resfriamento, equação (3.9)

$a_{i} \quad$ Coeficientes da função, equação (12)

$b_{i} \quad$ Coeficientes da função, equação (13)

$B_{F}^{\alpha} \quad$ Taxa de exergia química associada ao fluxo de $\mathrm{kW}$ combustível através da superfície de controle

$B_{P}^{\alpha} \quad$ Taxa de exergia das saídas úteis $\mathrm{kW}$

$B_{Q}^{\complement} \quad$ Taxa de exergia associada à transferência de calor $\quad \mathrm{kW}$

$B_{W}^{\alpha} \quad$ Taxa de exergia associada à realização de trabalho $\quad \mathrm{kW}$

COP Coeficiente de desempenho

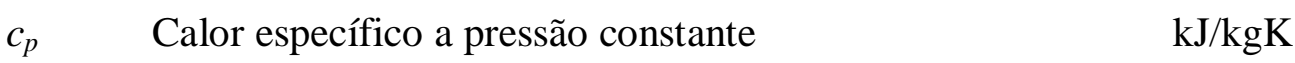

\& taxa de destruição de exergia $\quad \mathrm{kW}$

ECR razão de conversão de energia

I\& taxa de fornecimento de energia através do combustível $\mathrm{kW}$

$h \quad$ Entalpia específica $\quad \mathrm{kJ} / \mathrm{kg}$

\& taxa de irreversibilidade

n\& Vazão mássica $\mathrm{kg} / \mathrm{s}$

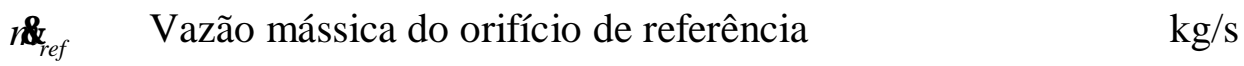

$P \quad$ Pressão absoluta bar

$q \quad$ Fração mássica de vapor ou título _ _

$£ \quad$ Taxa de transferência de calor $\quad \mathrm{kW}$

$\mathcal{Q}_{c} \quad$ Taxa de produção de frio ou potência frigorífica $\quad \mathrm{kW}$

$\oint_{n} \quad$ Taxa de calor de rejeito não aproveitado $\quad \mathrm{kW}$

$\oint_{r} \quad$ Taxa de calor de rejeito recuperado $\quad \mathrm{kW}$

$\mathcal{Q}_{g} \quad$ Taxa de ganhos de calor do ambiente (perdas) $\quad \mathrm{kW}$

$R \quad$ Razão entre as demandas de potência elétrica e de frio _ -

$T$ Temperatura absoluta K 
$\bar{T} \quad$ Temperatura média $\quad \mathrm{K}$

$\begin{array}{lll}s & \text { Entropia específica } \mathrm{kJ} / \mathrm{kgK}\end{array}$

$s \quad$ Expoente dependente do grau de sub-resfriamento, equação (3.9)

Taxa de trabalho fornecido ou potência elétrica kW

$W_{\max }^{\mathscr{Q}}$ Máxima potência realizada por um motor primário $\mathrm{kW}$

$v \quad$ Volume específico $\quad \mathrm{m}^{3} / \mathrm{kg}$

$x \quad$ Concentração de amônia —

\section{Símbolos gregos}

$\alpha$ Razão de recuperação de energia do grupo motor térmico/gerador elétrico.

$\beta \quad$ Razão de distribuição de produção de frio entre os chillers de absorção e de compressão de vapor

$\gamma \quad$ Razão percentual de demanda de potência elétrica.

$\delta \quad$ Defeito de eficiência

$\Delta \quad$ Variação

$\varepsilon \quad$ Efetividade

$\varepsilon_{m} \quad$ Efetividade da transferência de massa

$\varepsilon_{h} \quad$ Efetividade da transferência de calor

$\eta \quad$ Eficiência

$\theta \quad$ Coeficiente de perdas

$\lambda \quad$ Razão de distribuição da carga entre os motores primários

$\mu \quad$ Concentração de etilenoglicol no circuito de água gelada

$\varphi \quad$ Coeficiente de exergia de combustível

$\Phi \quad$ Relação de pressões

$\chi \quad$ Razão de carga parcial do motor primário

$\psi \quad$ Eficiência racional 


\section{Sub-índices e abreviaturas}

\begin{tabular}{|c|c|}
\hline$A C$ & Chiller de absorção \\
\hline$C$ & Refrigeração \\
\hline$C O N$ & Condensador \\
\hline$c w$ & Água gelada \\
\hline$D E$ & Demanda \\
\hline$e$ & Mistura água/etileno-glicol \\
\hline$E G$ & Gerador elétrico \\
\hline$E V A$ & Evaporador \\
\hline$g$ & Ganho de calor \\
\hline$G B$ & Volume de controle englobando toda a planta \\
\hline$G E N$ & Gerador \\
\hline$H S$ & Fonte de calor \\
\hline$h w$ & Água quente \\
\hline$i$ & Componente \\
\hline$I C$ & Motor de combustão interna \\
\hline in & Entrada \\
\hline $\lim$ & Limite \\
\hline $\max$ & Máximo \\
\hline $\min$ & Mínimo \\
\hline$n$ & Não recuperado \\
\hline nom & Nominal \\
\hline out & Saída \\
\hline$P U M P$ & Bomba \\
\hline$Q c$ & Relativo à demanda de frio \\
\hline$r$ & Recuperado \\
\hline$R E C$ & Retificador \\
\hline$R H E$ & Trocador regenerador interno \\
\hline$R E J$ & Rejeitado \\
\hline sat & Saturação \\
\hline$s c$ & Sub-resfriamento \\
\hline
\end{tabular}


SCA Absorvedor regenerador (Resfriado pela solução forte) Fonte de calor

TO Total dos motores primários

VC Chiller de compressão de vapor

W Relativo à demanda de potência elétrica

w Água

WCA Absorvedor resfriado por água

$X$

Referente aos dados experimentais. 